\title{
Fabrication of a poly(dimethylsiloxane) microstructured surface imprinted from patterned silicon wafer with a self-cleaning property
}

\author{
Shingo Tamesue ${ }^{1,2}$, Eri Takahashi ${ }^{2}$, Shunsuke Kosugi ${ }^{2}$, Kazuhiro Fukami ${ }^{3}$, Tetsu Mitsumata ${ }^{1,2}$, \\ Norio Tsubokawa ${ }^{1}$, Tetsuo Sakka ${ }^{4}$ and Takeshi Yamauchi ${ }^{1,2}$
}

Polymer Journal (2016) 48, 835-838; doi:10.1038/pj.2016.34; published online 23 March 2016

\section{INTRODUCTION}

Poly(dimethylsiloxane) (PDMS) is used in many fields, such as automobiles, cosmetics and other industries, because of its thermal stability, optical transparency, mechanical strength and biocompatibility. However, one disadvantage is the difficulty in removing stains from its surface. Clues to solve this previously reported problem include development of the monomer and treating the material with plasma treatment processes. ${ }^{1-3}$ These approaches exhibit great potential for generating surfaces from which strains can be easily removed; however, manufacturing processes for such approaches can be complicated and expensive.

Nature provides a wide selection of surfaces through microstructures, such as a leaf's surface ${ }^{4}$ and a gecko's foot hair ${ }^{5}$. Lotus leaves ${ }^{6}$ and rose petals ${ }^{7}$ also exhibit microstructures on their surfaces as well as superhydrophobicity, which is demonstrated through contact angles higher than $150^{\circ}$. However, these superhydrophobic surfaces exhibit different levels of contact angle hysteresis, which is the difference between an advancing contact angle and a receding contact angle. ${ }^{8}$ Lotus leaf surfaces feature low contact angle hysteresis on which a water droplet rolls down the surface with slight tilt. ${ }^{9}$ On the other hand, the rose petal surface exhibits a high water contact angle; it exhibits strong water droplet adhesion and a pinning property that has been attributed to van der Waals interactions and capillary force. These phenomena are referred to as a 'lotus effect' and 'petal effect', respectively, and these effects facilitate self-cleaning. The lotus effect results in self-cleaning because the water droplets roll easily across the surface and pick up the stains on their way. ${ }^{10}$ The petal effect causes self-cleaning because the water droplets are pinned to the surface and repel oil. ${ }^{11}$
Previously, researchers reported that PDMS with a microstructure exhibited a self-cleaning property. ${ }^{12}$ For practical use of PDMS with a self-cleaning property, the manufacturing cost is important. If we can print a PDMS microstructure with a self-cleaning property from a mold, the manufacturing effort and cost will markedly decrease. However, the literature only contains a few examples of PDMS with a microstructure surface imprinted from molds that exhibit a self-cleaning property. ${ }^{13}$

In this study, we newly fabricated a microstructure on a PDMS surface using a porous silicon wafer as a mold. We expected that the PDMS microstructure surface performs self-cleaning similar to the lotus and petal effects.

A porous silicon wafer can be easily controlled through tuning the parameters, such as pitch size and depth, which were precisely determined using a prepattern, an etching solution, the current density and the etching time. ${ }^{14,15}$ In principle, porous silicon is uniformly prepared on a whole wafer surface with several inches in diameter. ${ }^{16}$ This construction is strongly advantageous for large-scale production of polymer films with tailored surface morphologies. Therefore, this approach may be one of the most highly effective, simple and convenient methods for fabricating a microstructure on various surfaces over large-scale areas. Additionally, we expected that the fabrication cost will be low because microrod surfaces can be easily reproduced using a porous silicon wafer as the mold without other chemicals and techniques.

\section{EXPERIMENTAL PROCEDURE}

The microstructured PDMS was prepared as shown in Figure 1. We prepared the silicon wafer microstructured surface in accordance with the Supplementary Information, and we coated the surface with a mold release agent containing

${ }^{1}$ Department of Material Science and Technology, Faculty of Engineering, Niigata University, Niigata, Japan; ${ }^{2}$ Graduate School of Science and Technology, Niigata University, Niigata, Japan; ${ }^{3}$ Department of Material Science and Engineering, Graduate School of Engineering, Kyoto University, Kyoto, Japan and ${ }^{4}$ Department of Energy and Hydrocarbon Chemistry, Graduate School of Engineering, Kyoto University, Kyoto, Japan

Correspondence: Dr S Tamesue, Department of Material Science and Technology, Faculty of Engineering, Niigata University, Ikarashi 2-8050, Nishi-ku, Niigata 950-2181, Japan. E-mail: tamesue@eng.niigata-u.ac.jp

or Dr K Fukami, Department of Material Science and Technology, Kyoto University, Kyoto 606-8501, Japan.

E-mail: fukami.kazuhiro.2u@kyoto-u.ac.jp

or Dr T Yamauchi, Department of Material Science and Technology, Faculty of Engineering, Niigata University, Ikarashi 2-8050, Nishi-ku, Niigata 950-2181, Japan.

E-mail: yamauchi@gs.niigata-u.ac.jp

Received 11 November 2015; revised 27 January 2016; accepted 28 January 2016; published online 23 March 2016 


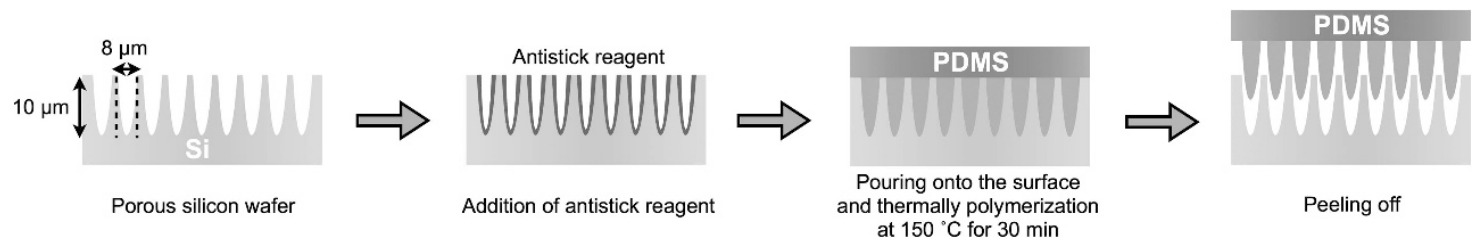

Figure 1 Preparing the poly(dimethylsiloxane) (PDMS) surface with a microrod structure by transcribing the silicon wafer microstructure. A full color version of this figure is available at the Polymer Journal journal online.
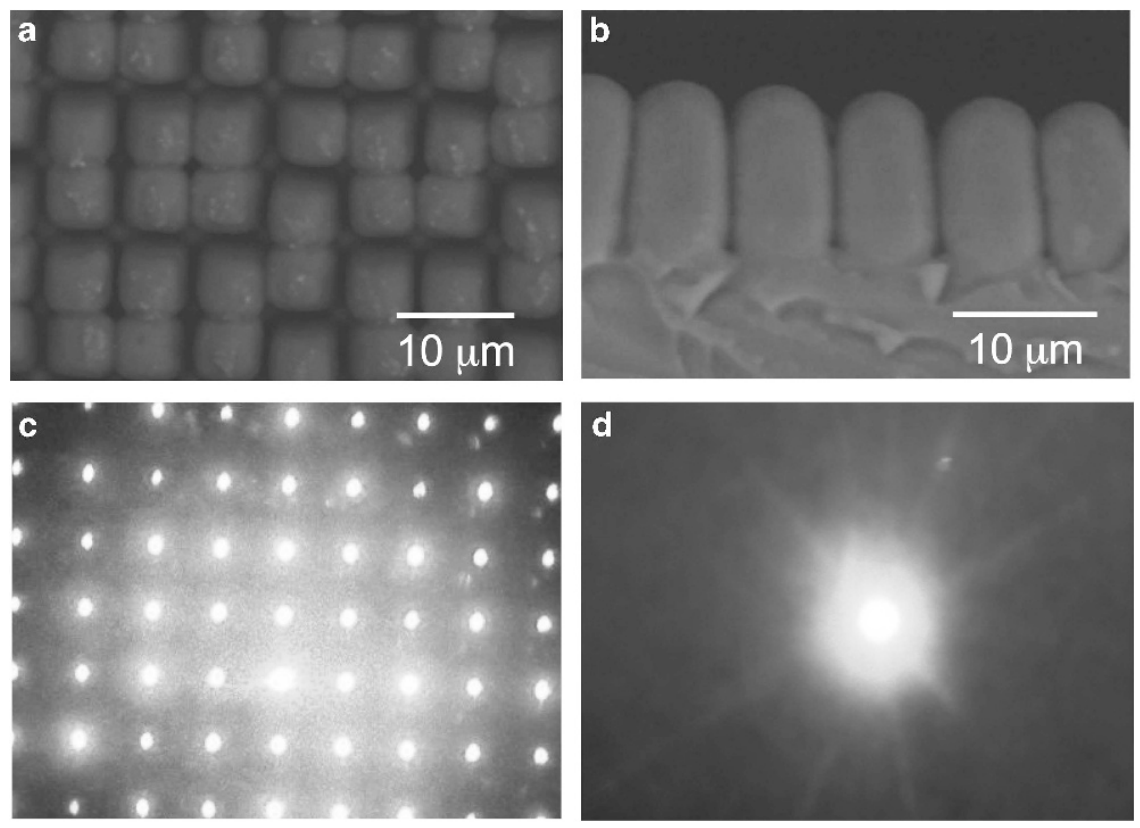

Figure 2 Scanning electron microscopy (SEM) images of the microstructured poly(dimethylsiloxane) (PDMS) surface: (a) top view and (b) side view. Photograph of transmitted light through (c) the microstructured PDMS and (d) the flat PDMS. A full color version of this figure is available at the Polymer Journal journal online.

water/detergent/olefin at a 18:2:1 weight ratio. After fabricating the mold, the PDMS prepolymer and a curing agent were thoroughly mixed at a 10:1 weight ratio using a mixer, which was poured onto the mold and cured at $150^{\circ} \mathrm{C}$ for $30 \mathrm{~min}$ in an oven. Finally, the microstructured PDMS replica was obtained by peeling the PDMS off of the mold.

\section{RESULTS AND DISCUSSION}

The microstructured PDMS surfaces were deposited with gold and imaged using scanning electron microscopy (SEM) as shown in Figures $2 \mathrm{a}$ and $\mathrm{b}$. As shown, we confirmed that the microrod structure corresponds to the porous silicon wafer formed on the surface. The average height and width of the microrods measured using scanning electron microscopy imaging were 10 and $8 \mu \mathrm{m}$, respectively. Hence, we confirmed that the microrod structure was printed onto the PDMS from the silicon wafer because the microrod shape was consistent with the mold shape. Thus, we fabricated PDMS with regularly arranged microrods.

Figures $2 \mathrm{c}$ and $\mathrm{d}$ show photographs of the transmitted light patterns through the microstructured PDMS and flat PDMS detailed in Supplementary Figure S1. As shown in Figure 2d, the pattern through the flat surface was a single point. On the other hand, the pattern for the microstructure surface was regularly arranged as shown in Figure $2 \mathrm{c}$. The pattern is attributed to the diffraction behavior of a

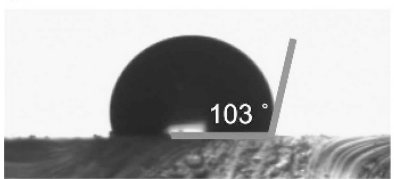

b

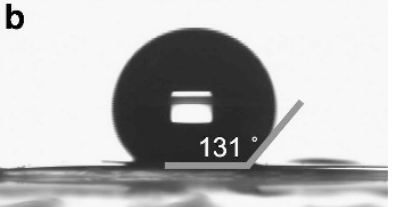

Figure 3 Water droplet contact angles on (a) a flat surface and (b) a microstructured surface of poly(dimethylsiloxane) (PDMS). A full color version of this figure is available at the Polymer Journal journal online.

the microrod structure. Furthermore, the theoretical rod intervals were determined using Bragg's equation as $d_{t}$, and the measuring rod intervals were determined using an optical microscope as $d$; the intervals were compared. We estimated $d_{\mathrm{t}}$ and $d$ as 8.51 and $8.22 \mu \mathrm{m}$, respectively. Therefore, we believe that the fabricated PDMS features microrods with the same sizes arranged both horizontally and vertically.

The PDMS water contact angles were measured and calculated as shown in Figure 3. The results show that the water contact angle was $103^{\circ}$ on the flat surface and $131^{\circ}$ on the microstructured surface; hence, the microstructure increased hydrophobicity. The effect of the surface roughness on wettability was theoretically modeled using two standard approaches, namely the Wenzel model ${ }^{17}$ and the 
Cassie-Baxter model ${ }^{18}$ (Supplementary Figure S2). These models for square-type pillar geometries ${ }^{19}$ were used to interpret the aforementioned results.

Wenzel assumed that liquid completely fills the depression in the region that contacts the substrate. The contact angle, $\theta_{\mathrm{w}}$, is given by the following equation:

$$
\cos \left(\theta_{\mathrm{w}}\right)=r \cos \left(\theta_{\mathrm{Y}}\right)
$$

where $r$ is the ratio of the actual area of the rough surface to the projected area and $\theta_{\mathrm{Y}}$ is the intrinsic contact angle on a flat surface of the same nature as the rough surface, which is referred to as Young's contact angle. The contact angle of the silicone rubber deposited on a flat substrate was measured at $103^{\circ}$, and this value was used for the theoretical calculations.

In contrast, Cassie-Baxter assumed that a composite surface forms when a droplet contacts a rough surface, and a droplet is completely lifted up by the roughness features. The apparent contact angle, $\theta_{\mathrm{CB}}$, is given by the following equation:

$$
\cos \left(\theta_{\mathrm{CB}}\right)=f_{\mathrm{SL}}\left[\cos \left(\theta_{\mathrm{Y}}\right)+1\right]-1
$$

where $f_{\mathrm{SL}}$ is the fractional geometrical area of the solid-liquid interface under the drop.

For the pillar geometries used in this work, $r$ and $f_{\mathrm{SL}}$ can be written as follows:

$$
\begin{aligned}
& r=\frac{(a+b)^{2}+4 H a}{(a+b)^{2}} \\
& f_{\mathrm{SL}}=\frac{4 a^{2}}{4 a^{2}+4 a b+b^{2}}
\end{aligned}
$$

where $a$ is the side of the square-shaped pillar, $H$ is the height of the pillar and $b$ is the interpillar distance. Both theoretical contact angles, $\theta_{\mathrm{W}}$ and $\theta_{\mathrm{CB}}$, were calculated from the aforementioned theoretical models using Equations (1)-(4) and are $106^{\circ}$. The experimental value was greater than the theoretical values likely because the shape of the transcribed microrod is not entirely quadrangular and is more or less round as in the theoretical model shown in Figures $2 \mathrm{a}$ and $\mathrm{b}$.

To study the effect of microstructures with different pitch values on the contact angles, we fabricated PDMS microstructure surfaces with 3 and $5 \mu \mathrm{m}$ pitches and compared the experimental and theoretical values. Supplementary Table S1 and Supplementary Figure S3a summarize the measured and calculated contact angles in the Wenzel and Cassie-Baxer models. As shown in Supplementary Table S1, the experimental values decreased as the pitch values decreased, and the experimental value for the microrod surface with a 3- $\mu \mathrm{m}$ pitch was consistent with the theoretical value using the Cassie-Baxter model. As shown in Supplementary Figure S3b, the microrod structure shape became more similar to the theoretical model as the pitch size decreased, and the water droplets could not fit into the slit between the microrods; this microstructure exhibited Cassie-Baxter-type wetting behavior.

The contact angles for various oil compounds and water increased with the microrod structure as shown in Table 1 . The wetting contact line likely remains pinned at the microstructure. In contrast, $n$-hexadecane showed a lower contact angle on the microstructured surface compared with the flat surface. We suspect that the microstructured surface pinned highly viscous liquid, but low viscous liquid could spread. Thus, we confirmed that surface characteristics changed because of the microrod structure transcribed from the patterned silicon wafer.
Table 1 Contact angles of organic solvents on the flat and mirostructured PDMS

\begin{tabular}{lrc}
\hline & \multicolumn{2}{c}{ Contact angle/deg. } \\
\cline { 2 - 3 } Organic solvent & Flat & Microstructured \\
\hline Ethyleneglycol & 100 & 126 \\
Glycerol & 94 & 127 \\
Dimethylsulfoxide & 90 & 119 \\
Propylenecarbonate & 89 & 109 \\
Olive oil & 54 & 63 \\
Silicone oil & 0 & 15 \\
$n$-Hexadecane & 38 & 7 \\
\hline
\end{tabular}

Abbreviation: PDMS, poly(dimethylsiloxane).

The adhesive force of each PDMS sample was evaluated by tilting the sample to $90^{\circ}$. On the flat surface, when the droplet size reached $7.0 \mu$ l, water droplets began flowing (Supplementary Figure S4). In contrast, on the microstructure surface, water droplets began flowing when the droplet size reached $24.0 \mu \mathrm{l}$. We used the results to calculate the adhesive force using Supplementary Equation (S2). The calculated adhesive forces on the flat surface and microstructured surface were 7.0 and $24.0 \mu \mathrm{N}$, respectively. Researchers reported that natural rose petal exhibits a $63.8 \mu \mathrm{N}$ adhesive force. ${ }^{20}$ Hence, the PDMS microstructured surface exhibits a high adhesive force; however, the force is lower than the rose petal.

Supplementary Figure S5 shows the effect of droplet mass on the sliding angle. A 7- $\mu \mathrm{l}$ water droplet flowed on the flat surface, and the sliding angle decreased with an increase in the water droplet mass. However, a $24-\mu$ l water droplet remained pinned on the microstructure surface, and the sliding angle decreased slowly with an increase in the water droplet mass. Supplementary Figure S6 shows the sliding behavior of a 25.0- $\mu$ l water droplet on the flat surface and the microstructure surface. On the flat surface, the water droplet slid easily when the sample was tilted. In contrast, the microstructure surface retained the water droplet. Thus, we found that the PDMS microstructure surface exhibits a higher adhesive force than the flat surface; the water likely penetrates the microstructure similar to a rose petal.

Figure 4 shows the PDMS microstructure surface before and after the self-cleaning test detailed in Supplementary Figure S7 using silicon carbide ( $\mathrm{SiC}$ ) as a stain model. As shown in Figure 4, particles were deposited evenly before the self-cleaning test. After rinsing with water, the $\mathrm{SiC}$ particles remained on the flat surface. In contrast, the $\mathrm{SiC}$ particles on the microrod surface clearly decreased. Supplementary Table S2 shows the remaining particle area before and after the self-cleaning test. While $\mathrm{SiC}$ particles remained on the flat surface area, many $\mathrm{SiC}$ particles were removed from the microstructured surface area. Furthermore, wide microscope images of SiC particles on the surfaces at different microrod pitches $(3,5$ and $8 \mu \mathrm{m})$ are shown in Supplementary Figure S8. Further, their SiC particle rejection ratios were calculated based on Supplementary Figure S8 after the self-cleaning test shown in Supplementary Figure S9, which indicates that the surface with a wider microrod pitch exhibited a superior self-cleaning property. These results clearly show a correlation with the water droplet contact angles on the different pitch surfaces in Supplementary Table S1 and Supplementary Figure S3. Based on these results, we confirm that the $\mathrm{SiC}$ particles can be removed more easily from the microstructured surface with the higher contact angle for water and oil droplets because water droplets wash out the $\mathrm{SiC}$ 

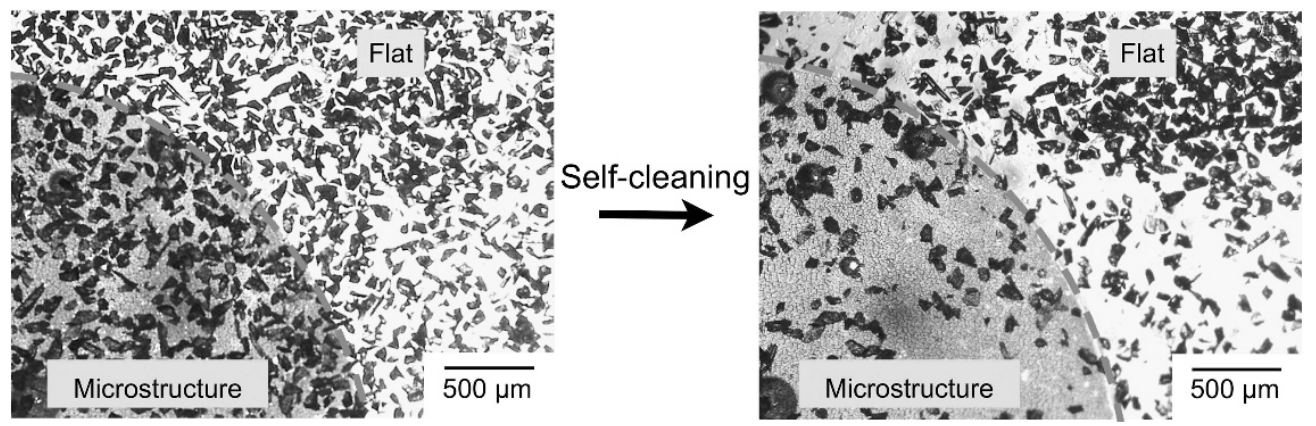

Figure 4 Self-cleaning test using the poly(dimethylsiloxane) (PDMS) and silicon carbide (SiC) as the theoretical contamination. The area on the left side of the dashed line shows the microstructured surface area, and the area on the right side of the dashed line shows the flat surface area. A full color version of this figure is available at the Polymer Journal journal online.

particles more easily. Thus, the microstructured PDMS surface gained the self-cleaning property because of its high contact angle with oil and water.

\section{CONCLUSION}

In summary, we easily fabricated a microstructured PDMS by transcribing the silicon wafer surface structure with controllable pore sizes. The microstructure surface exhibited clear hydrophobicity and oleophobicity. Based on theoretical and experimental results, we found that microstructures enhance hydrophobicity by increasing the surface roughness based on the Cassie-Baxter model. The adhesive force was sufficiently strong to capture water even when the surface was upside down. The SiC particles on the microstructured surface were removed owing to water droplet penetration of the microstructure. This self-cleaning property would not only benefit applications in the medical field, such as for contact lenses, but also apply to surfaces that require self-cleaning, such as architectural material and cookware.

\section{CONFLICT OF INTEREST}

The authors declare no conflict of interest.

\section{ACKNOWLEDGEMENTS}

We appreciate Ms Kumi Hashimoto and Mr Takeshi Fukuda for their kind help and support. This work was supported by JSPS KAKENHI, Grant Number 2412001.

1 Nie, Z. \& Kumacheca, E. Patterning surfaces with functional polymers. Nat. Mater. 7, 277-288 (2008).

2 Zdyrko, B., Klep, V. \& Luzinov, I. Universal platform for surface modification employing grafted polymer layers. Mater. Matt. 3, 44 (2008).
3 Nagata, H., Ko, S. W., Randall, C. A. \& Trolier-McKinstry, S. Microcontact printed $\mathrm{BaTiO}_{3}$ and $\mathrm{LaNiO}_{3}$ thin films for capacitors. J. Am. Ceram. Soc. 89, 2816-2821 (2006).

4 Kesong, L. \& Lei, J. Bio-inspired design of multiscale structures for function integration. Nano Today 161, 155-175 (2011).

5 Hansen, W. R. \& Autumn, K. Evidence for self-cleaning in gecko setae. Proc. Natl Acad. Sci. USA 102, 385-389 (2005).

6 Marmur, A. The lotus effect: superhydrophobicity and metastability. Langmuir 20, 3517-3519 (2004).

7 Feng, L., Zhang, Y., Xi, J., Zhu, Y., Wang, N., Xia, F. \& Jiang, L. Petal effect: a superhydrophobic state with high adhesive force. Langmuir 24, 4114-4119 (2008)

$8 \mathrm{Xi}$, J. \& Jiang, L. Biomimic superhydrophobic surface with high adhesive forces. Ind. Eng. Chem. Res. 47, 6354-6357 (2008).

9 Koch, K. \& Barthlott, W. Superhydrophobic and superhydrophilic plant surfaces: an inspiration for biomimetic materials. Philos. Trans. R. Soc. A 367, 1487-1509 (2009).

10 Barthlott, W. \& Neinhuis, C. Purity of the sacred lotus, or escape from contamination in biological surfaces. Planta 202, 1-8 (1997).

11 Shunsuke, N. \& Bharat, B. Bioinspired self-cleaning surfaces with superhydrophobicity, superoleophobicity, and superhydrophilicity. RCS Adv. 3, 671-690 (2013).

12 Park, E. J., Yoon, H. S., Kim, D. H., Kim, Y. H. \& Kim, Y. D. Preparation of self-cleaning surfaces with a dual functionality of superhydrophobicity and photocatalytic activity. Appl. Surf. Sci. 319, 367-371 (2014).

13 Cho, W. K. \& Choi, I. S. Fabrication of hairy polymeric films inspired by Geckos: wetting and high adhesion properties. Adv. Funct. Mater. 18, 1089-1096 (2008).

14 Fukami, K., Harraz, F. A., Yamauchi, T., Sakka, T. \& Ogata, Y. Fine-tuning in size and surface morphology of rod-shaped polypyrrole using porous silicon as template. Electrochem. Commun. 10, 56-60 (2008).

15 Okayama, F., Fukami, K., Plugaru, R., Sakka, T. \& Ogata, Y. Ordering and disordering of macropores formed in prepatterned p-type silicon. J. Electrochem. Soc. 157, D54-D59 (2010).

16 Hossain, S. M., Das, J., Chakraborty, S., Dutta, S. K. \& Saha, H. Electrode design and planer uniformity of anodically etched large area porous silicon. Semicond. Sci. Technol. 17, 55-59 (2002).

17 Wenzel, R. N. Resistance of solid surfaces to wetting by water. Ind. Eng. Chem. 28, 988-994 (1936).

18 Cassie, A. B. D. \& Baxter, S. Wettability of porous surfaces. Trans. Faraday Soc. 40, 546-551 (1944).

19 Athanasios, M., Roberto, G., Ilker, S. B., Evie, L. P., Roberta, R., Michele, M. \& Athanassia, A. Self-cleaning organic/inorganic photo-sensors. ACS Appl. Mater. Interfaces 5, 7139-7145 (2013)

20 Law, J. B. K., Ng, A. M. H., He, A. Y. \& Low, H. Y. Bioinspired ultrahigh water pinning nanostructures. Langmuir 30, 325-331 (2014).

Supplementary Information accompanies the paper on Polymer Journal website (http://www.nature.com/pj) 